\title{
Numerical Simulation of the Influence of Vaccines on Covid-19 Control: A Warning for Its Spread and Control
}

\section{Moises Meza Pariona, \& Luan Daniel Pelotoni}

State University of Ponta Grossa, Dpts Materials Engineering and Mathematic and Statistic, Ponta Grossa-PR, Brazil

DOI - http://doi.org/10.37502/IJSMR.2021.4808

\begin{abstract}
The goal of this study was to apply a modified susceptible-exposed-infectious-recovered (SEIR) compartmental mathematical model for prediction of COVID-19 epidemic dynamics, for the high and low pandemic case, which occurred during the year 2021 at State de Paraná Brazil and these results were compared. For this procedure, the model parameters using officially reported of State de Paraná Brazil were calibrate. As result the $\mathrm{S}$ (susceptible population) and $\mathrm{E}$ (exposed population) parameters decay as a function of time, being a very drastic drop for $\mathrm{S}$ and a slow decrease to $\mathrm{E}$ in high pandemic, however, the I (infected population) parameter rises and decays as a function of time, but, in a high pandemic, the tendency is to grow, nevertheless, the $\mathrm{R}$ (recovered population) parameter rises as a function of time, in low pandemics this parameter has a much higher growth behavior than in high pandemics, as expected. So, the numerical simulation is consistent with reality. This result is coherent with what is happening in the scenario in the different cities of the countries of the world. Among the advantages of the implemented model, it should be noted that despite the simplicity of the hypotheses, the adjustments obtained were quite accurate and the projections made do not differ much from those other more complex models. Our results could also provide useful suggestions for the prevention and control of the COVID-19 outbreaks in different countries and locations.
\end{abstract}

Key-word: Covid-19 epidemic, epidemiological models, vaccine, SEIR model, spread and control, numerical simulation.

\section{Introduction}

The new and highly contagious coronavirus disease 2019 (COVID-19) caused by SARS-CoV-2 (severe acute respiratory syndrome-coronavirus 2) exploded worldwide since early December 2019, resulting 4.367.023 deaths and 207.557.334 infected in the world, in Brazil 569.058 deaths until August 16, 2021, from COVID-19 Data Report from the Center for Systems Science and Engineering (CCSE) at Johns Hopkins University 2021 [1]. According to the Paraná Health Secretariat [2], at September 13, 2021, there were 20.999.779 infected and 586.851 deaths in the country; 1.473.335 infected and 37.896 deaths at State of Paraná, Brazil. For countries around the world have taken various control measures to mitigate the spread of 
the virus, including social distancing measures, wearing masks, and so on. In the last few months its spread has increased dramatically, leading to hospital bed shortages.

Healthcare professionals and public health authorities have a central role in vaccination against COVID-19 of the people. Vaccines play a critical role in preventing deaths, hospitalization caused by infectious diseases. Emerging data on effectiveness indicates that licensed COVID-19 vaccines are contributing to controlling the spread of the disease. Until widespread vaccination has been achieved, both vaccinated and unvaccinated people need to be aware of the additional protective behaviors required to control the pandemic locally, according to World Health Organization [3].

Vaccination has been shown to contribute to reducing deaths and severe illness from COVID-19, and to reduce the transmission of COVID-19. Vaccinating as many people as possible and reducing the spread of disease is important. Vaccination of a significant proportion of the population also protects vulnerable people, including those who cannot receive vaccines, or the small proportion of people who might remain at risk of infection after vaccination. Failure to vaccinate widely also enables continued circulation of the virus and the generation of variants, including some that may pose a greater risk. Widespread vaccination will help prevent people from having to go to hospital and contribute to fewer people getting sick, ultimately alleviating the burden of COVID-19 on healthcare systems. It will also help allow a return to normal societal functioning and the re-opening of economies, as was reported [3].

This paper aims to shows the advantage of mathematical modeling and their simulations in times like now, during which the COVID-19 spreading and the dynamics of epidemic transmission was studied, for the high and low pandemic case that occurred during the year 2021 and these results were compared, for this the Susceptible-Exposed-Infectious-Removed (SEIR) model was used. These models are based on a system of ordinary differential equations. This research studied the evolution of the parameters as a function of time for the COVID-19 epidemic, which can be applied in any city.

\section{Mathematical Modeling}

The 2019 coronavirus disease (COVID-19) seriously threatened human lives and health worldwide. Many epidemic models have been developed to provide as references for decision making by rulers and the World Health Organization. To run and understand the characteristics of the epidemic trend, parameter optimization algorithms are needed to obtain the model mechanisms.

In the considered transmission model total human population is divided into four sub compartments, where $S$ denotes susceptible population, which represent the section of human population who are susceptible to contact the virus and become infectious if exposed. $E$ denotes exposed population, which represent human population who are infected but not infectious so far or suspect. I denote infected population, who have fully developed the symptoms of COVID-19 and can spread the virus through contact with the susceptible population. The populations section who has fully recovered after the treatment and they have no symptoms of disease (free from the disease) is denoted by $R$. To respect was presented a discussed by different authors, among them, Yadava and Verma [4], Savioli et al. [5], Carcione et al. [6], Lopez and Rodo [7], Din and Algehyne [8], Cintra and Fontinele [9]. 
The transmission model for COVID-19 pandemic is given by following system of ordinary differential equations;

$$
\begin{aligned}
& \frac{d S}{d t}=-\frac{\beta S I}{N} \\
& \frac{d E}{d t}=\frac{\beta S I}{N}-a E \\
& \frac{d I}{d t}=a E-\gamma I \\
& \frac{d R}{d t}=\gamma I
\end{aligned}
$$

In the dynamical system of equations, the rate of human-to-human transmission of disease between the exposed and susceptible individuals are given by the function $\beta$, the rate of human-to-human transmission of disease between the infected and susceptible individuals are denoted by the function $\alpha$ and the average disease incubation period is denoted by $\alpha^{-1}$ and whereas $\gamma$ denote the rate of recovery and infectious period is $\gamma^{-1}$. In Figure 1, a schematic diagram is shown the transmission model for COVID-19 pandemic.

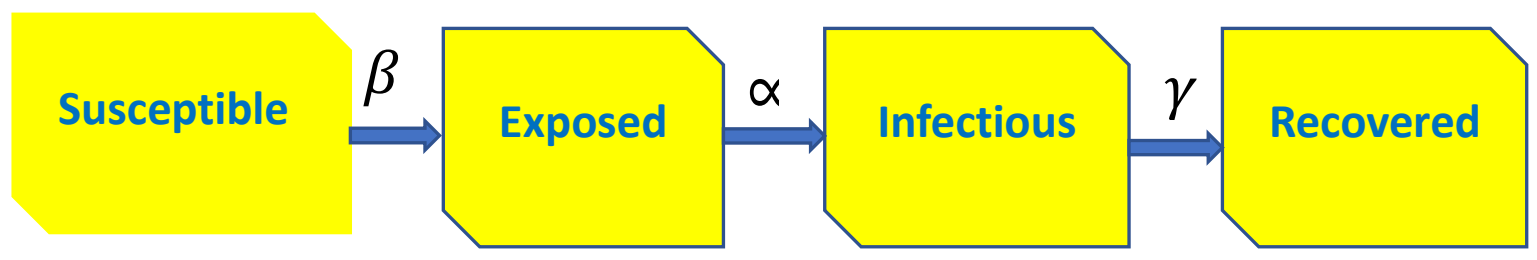

Figure 1. A schematic diagram showing the transmission model for COVID-19 pandemic.

An important parameter in any epidemic, what is called the basal reproduction rate or the basic reproduction ratio, $\mathrm{R}_{\mathrm{t}}$, is the classical epidemiological measure associated with the reproductive power of the disease, defined as the growth rate times the time of infection, Carcione et al. [6]:

$$
\mathrm{R}_{\mathrm{t}}=\mathrm{b} / \alpha
$$

Therefore, if $R_{t}>1$, there will be exponential growth of the epidemic at the beginning of the contagious process. $R_{t}<1$, there will be attenuation also exponential, until the epidemic ends. Therefore, $\mathrm{R}_{\mathrm{t}}$ is a fundamental parameter for epidemic control.

To do this work, the effectiveness of vaccines in the state of Paraná Brazil in the year 2021 was studied by numerical simulation, comparing the high contamination that occurred in the month of February, where the vaccination percentage was low, and ta the month of September had low contamination and a high percentage of vaccinated people. In this work, in order to carry out the simulation, we considered the data according to the Paraná Health Department [2] and Ministério da Saúde Secretaria de Vigilância em Saúde [10].

Thus, the data corresponding to the month of February 20, 2021. Where, $\mathrm{I}_{\mathrm{o}}$ (as confirmed) 607.632 , initial status of those infected. $\mathrm{R}_{\mathrm{o}}$, recovered 449.980 , initial condition of those recovered. $\mathrm{E}_{\mathrm{o}}$ (as notified or suspect) 1.529.476, initial condition of those exposed. $\mathrm{N}=$ 11.516.840, population number of the State of Paraná. Thus, $\mathrm{So}=\mathrm{N}-\mathrm{E}_{\mathrm{o}}-\mathrm{I}_{\mathrm{o}}-\mathrm{R}_{\mathrm{o}}=8.929 .752$, initial condition of the susceptible. The baseline reproduction index, $R_{t}=1.5, \alpha=1 / 5.2\left[\right.$ day $^{-1}$ ], as $R_{t}$ $=\beta / \alpha$, so $\beta=1.5 / 5.2\left[\right.$ day $^{-1}$ ] and recovery rate $\gamma=1 / \mathrm{D}\left[\right.$ day $^{-1}$ ], according to George [11], Lauer 
et. Al [12], Rees et al. [13], it was determined the average duration of recovery $\mathrm{D}$, the average of 10 days is used in this paper. To this date the vaccinated people were 8.006 .419 people, so the proportion vaccinated was $\delta=277.707 / \mathrm{N}=0.024$.

Meanwhile for the month of September 13, 2021. Where, $I_{0}$ (as confirmed) 1.473.335, initial status of those infected. $R_{0}$, recovered 1.382.448, initial condition of those recovered. $E_{0}$ (as notified or suspect) 5.005.082, initial condition of those exposed. $\mathrm{N}=11.516 .840$, population number of the State of Paraná. Thus, $\mathrm{So}=\mathrm{N}-\mathrm{E}_{0}-\mathrm{I}_{0}-\mathrm{R}_{0}=4.905 .975$, initial condition of the susceptible. The baseline reproduction index, $R_{t}=0.8, \alpha=1 / 5.2\left[\mathrm{day}^{-1}\right.$ ], as $R_{t}=\beta / \alpha$, so $\beta=0.8 / 5.2$ [day $^{-1}$ ]. To this date the vaccinated people were 8.006 .419 people, therefore the proportion vaccinated was $\delta=8.006 .419 / \mathrm{N}=0.695$, according to reports [2].

\section{Results and Discussions}

Next the result and discussion of the numerical simulation of the covid-19 epidemic through numerical simulation of epidemiological model will be presented, for this purpose the susceptible-exposed-infectious-removed (SEIR) model was used. We calibrate the model parameters using officially reported of State de Paraná Brazil, considering a state of pandemic high and low, in the State of Paraná Brazil [2].

The Equations 1-5 and all parameters involving these equations defined [8] in the previous section were used for this simulation. The simulation was done for the case when the population of the state of Paraná-Brazil showed a low vaccination percentage $(2.4 \%)$ in the month of February 2021 [2], and the result is shown in Figure 2 for 30 days. For this case the baseline reproduction index was $\mathrm{Rt}=1.5$ it was considered according to [2], which means a high contamination with a severe pandemic. It can be seen in Figure 2 that the susceptibility (S) has a drastic decrease as a function of time. However, the exposure (E) showed a very slow decline, after five days it tends to go up and then afterward 15 days it tends to go down slightly. Nevertheless, the infectious process (I) grows exponentially until it reaches a maximum in 25 days, soon thus decreasing slowly. Thus, through numerical simulation it is demonstrated a high infection during the high pandemic period and with a low vaccination percentage, which agrees with reality, where there was a lack of beds in hospitals and the population was forced to make look down. However, the recovery (R) grew with an almost linear behavior and having a high growth in relation to the contamination, which may have contributed to the percentage of vaccination and the look down. 


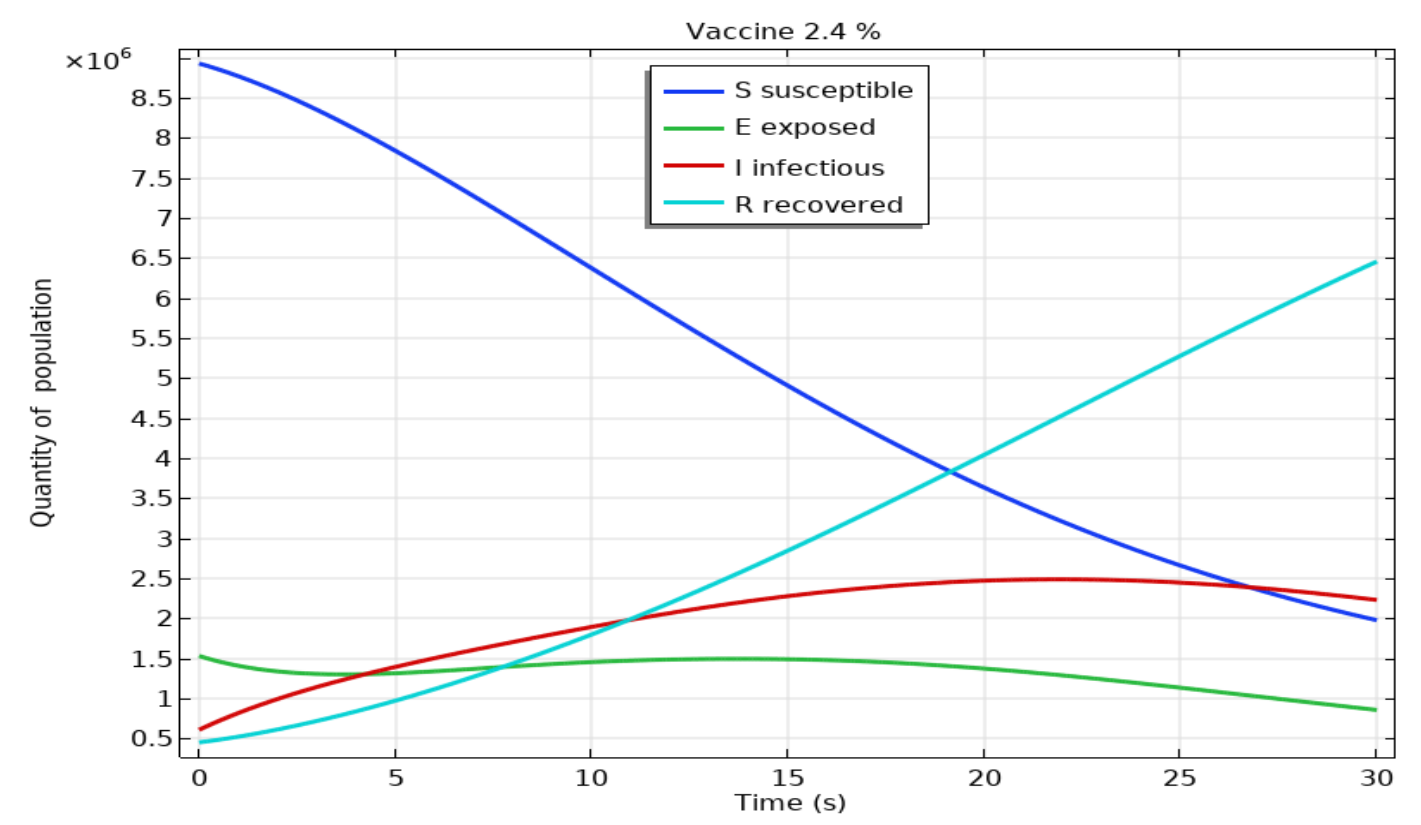

Figure 2. Numerical simulation of the covid-19 epidemic by SEIR model for the case with a severe pandemic

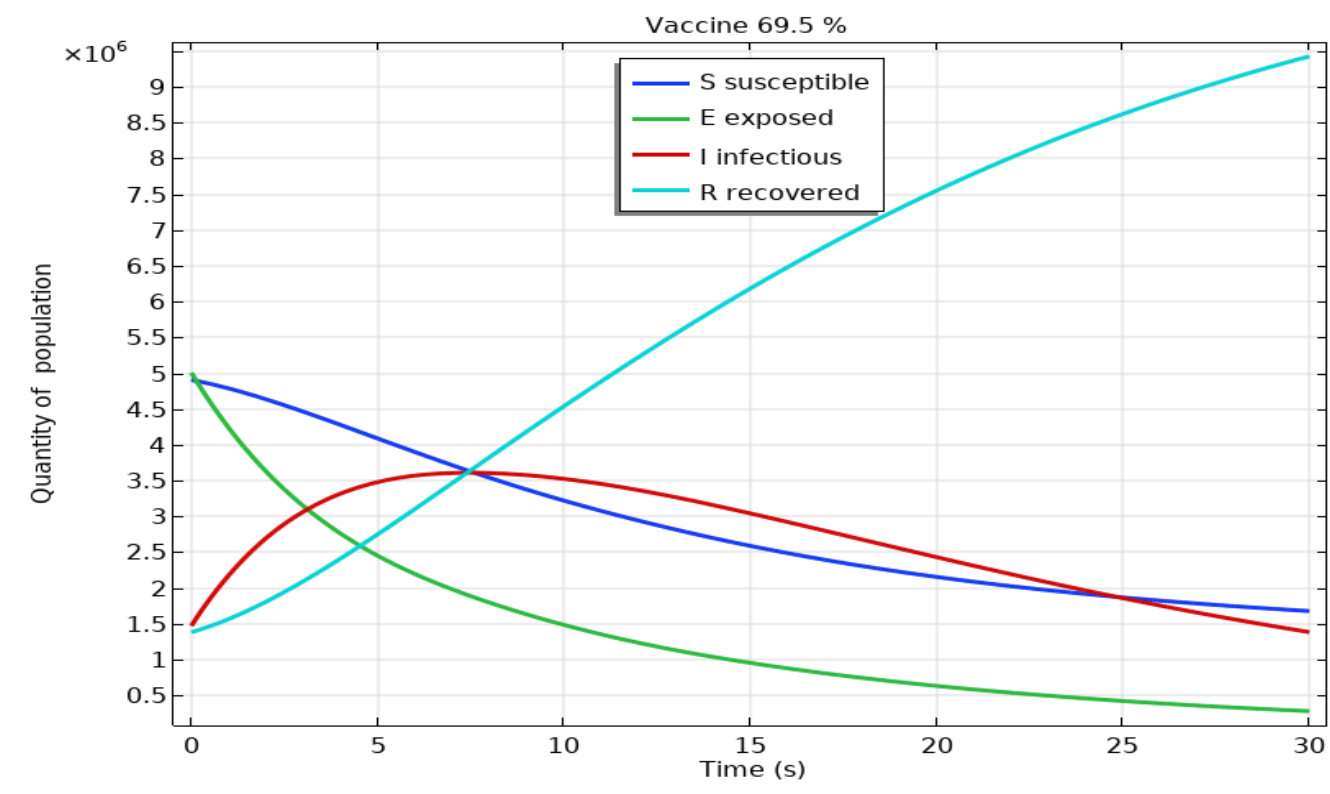

Figure 3. Numerical simulation of the covid-19 epidemic by SEIR model for the case with $69.5 \%$ vaccine

For the case with $69.5 \%$ vaccine, according to [2], where the baseline reproduction index was $\mathrm{R}_{\mathrm{t}}=0.8$, which means low contamination. The initial conditions for Figure 3 are completely different compared to Figure 2; in this last case these conditions are higher. The result can be seen in Figure 3, where the susceptibility (S) decays rapidly as a function of time, but this same parameter that corresponds to Figure 2, where the drop is drastic. In addition, the Exposure (E), also decays rapidly relative to $S$, but this drop is more abrupt relative to the same parameter of Figure 2. The infection parameter (I), rises quickly in the first days, then slowly decreases until the end of the period; this same parameter in Figure 2 with trend behavior to 
increase. This result is justified with the reality that is happening, with a high pandemic this last parameter its tendency is to grow, so the numerical simulation is consistent with the Covid19 event. Finally, the parameter recovery $(\mathrm{R})$ increases drastically, this behavior is much higher than in Figure 2, it means that at low pandemic the recovery is much higher, so the numerical simulation is consistent with reality.

The results of this work were similar to the authors, Yadava and Verma [4], Savioli et al. [5], Carcione and Santos [6], Lopez and Rodo [7]. The result presented is approximately similar to the data presented by the officially reported of State of Paraná Brazil [2]. To have a better closer of the simulation with the reality, the data provided by the health agencies would lack more accuracy, however, in this model the confinement, distancing, lockdown, the psychological, the emergence of transmutation of new viruses and emotional part were not considered. So, we will continue studied and sophisticate the model. Our challenge is to understand the mechanisms and the phenomenology of the process of emerged of new variants of coronavirus, spread, and control of the virus.

\section{Conclusions}

We calibrate the model parameters using officially reported of State de Paraná Brazil, considering a high and low pandemic for the covid-19 virus in this State.

In this result the $S$ (susceptible population) and $E$ (exposed population) parameters decay as a function of time, being a very drastic drop for $S$ and a slow decrease to $E$ in high pandemic. However, the I (infected population) parameter rises and decays as a function of time, but, in a high pandemic, the tendency is to grow. Nevertheless, the R (recovered population) parameter rises as a function of time, in low pandemics this parameter has a much higher growth behavior than in high pandemics as expected. So, the numerical simulation is consistent with reality.

This result is coherent with what is happening in the scenario of the different cities and countries of the world, due to a good percentage of people that were vaccinated, there is almost no more lockdown, besides that, schools, colleges, some universities and the employees of governmental companies are back in person, companies are returning to working normally, a certain amount of people are wearing less masks and more people are traveling. Because of this, our prediction from the covid-19 modeling is encouraging, less infection and more people recovering going on in the world.

Among the advantages of the implemented model, it should be noted that despite the simplicity of the hypotheses, the adjustments obtained were quite accurate and the projections made do not differ much from those other more complex models. Also, the instantaneous increment of cumulative diagnosed people depends on the history of cumulative infected people, by which the latent period can be taken into consideration.

Our results could also provide useful suggestions for the prevention and control of the COVID-19 outbreaks in different countries and locations

Results should be interpreted with care as projections at these stages of the epidemic are very dependent on the quality of data, with small changes in observed values producing large variations in trends. However, even with this limitation in mind, the magnitude of positive increments in cases suggests these results are strong. 
In general terms, the model is quite stable, as shown in the uncertainty analysis carried out. On the other hand, it can be seen in the successive updating of the parameters that the initial projections of the proposed model were not too far from the real data reported. This gives a special value to the work since it somehow justifies the previous discussion.

Models can be used to predict and understand how an infectious disease spreads in the world and how various factors affect the dynamics. Even if the predictions are inaccurate, it has been clear to scientists from many decades that quarantine, social distancing, and the adoption of very strict health and safety standards are essential to stop the spread of a virus. Quarantine was even implemented in medieval times to fight the Black Death before there was knowledge of the existence of viruses. In this sense, this pandemic reveals the failure of policymakers, since it is well-known from basic modeling results that earlier adoption of those measures can save thousands of lives and even prevent the pandemic. The interface of science, society, and politics is still uneasy, even in highly developed countries, revealing a disregard for scientific evidence. Moreover, one of the consequences is that some of these countries do not invest sufficiently in R\&D and must acquire the new technology from overseas at a much higher cost.

\section{References}

1) Johns Hopkins University (2021) COVID-19 Data Report from the Center for Systems Science and Engineering (CCSE) [Online]. Available: https://coronavirus.jhu.edu/map.html.

2) Paraná Health Secretariat (2021). Coronavírus (COVID-19). Secretaria da Saúde [Online]. Available: saude.pr.gov.br.

3) World Health Organization (2021) [Online]. Available. https://www.who.int/news/item/11-06-2021-statement-for-healthcare-professionalshow-covid-19-vaccines-are-regulated-for-safety-and-effectiveness

4) R.P. Yadava and R. Verma A numerical simulation of fractional order mathematical modeling of COVID-19 disease in case of Wuhan China. Chaos, Solitons and Fractals. vol. 140 pp. 110124, 2020. https://doi.org/10.1016/j.chaos.2020.110124

5) G. B. Savioli, J. M. Carcione, J. E. Santos, P M. Gauzellino, A. Ravecca, and A. Moras. A numerical simulation of the covid-19 epidemic in argentina using the seir model. Latin American Applied Research. vol. 51(3): pp.179-184, 2021. DOI:https://doi.org/10.52292/j.laar.2021.671

6) J. M. Carcione, J. E. Santos and J. A. Ba. Simulation of a COVID-19 Epidemic Based on a Deterministic SEIR Model. Front Public Health. v. 8, pp: 230, 2020. doi:10.3389/fpubh.2020.00230.

7) L. Lopez and X. Rodo. A modified SEIR model to predict the COVID-19 outbreak in Spain and Italy: Simulating control scenarios and multi-scale epidemics. Results in Physics. vol. 21, pp. 103746, 2021. https://doi.org/10.1016/j.rinp.2020.103746

8) R. U. Din, E. A. Algehyne. Mathematical analysis of COVID-19 by using SIR model with convex incidence rate. Results in Physics. vol. 23: 103970, 2021. https://doi.org/10.1016/j.rinp.2021.103970 
9) H. P. C. Cintra, F. N. Fontinele. Estimative of real number of infections by COVID-19 in Brazil and possible scenarios. Infectious Disease Modelling. vol. 5, pp. 720-736, 2020. https://doi.org/10.1016/j.idm.2020.09.004

10) Ministério da Saúde Secretaria de Vigilância em Saúde (2021) [Online]. Available: https://antigo.saude.gov.br/vigilancia-em-saude.

11) N. George, N. K. Tyagi, J. B. Prasad. COVID-19 pandemic and its average recovery time in Indian states. Clinical Epidemiology and Global Health. vol. 11, pp. 100740, 2021. https://doi.org/10.1016/j.cegh.2021.100740

12) S. A. Lauer et al. The incubation period of coronavirus disease 2019 (COVID-19) from publicly reported confirmed cases: estimation and application. Ann Intern Med. vol. 172(9), pp.577-582. 2020. https://doi.org/10.7326/M20-0504.

13) E. M. Rees, et al. COVID-19 length of hospital stay: a systematic review and data analysis, BMC Med. vol. 18(1), pp.1-14, 2020. https://doi.org/10.1186/s12916-02001726-3; 2020, 270. 